\title{
Akademisierte Pflegekräfte
}

\section{Potenziale besser nutzen}

— Mit der Akademisierung der Pflege sind in Deutschland Qualifikationsprofile entstanden, die die gesundheitliche Versorgung der Bevölkerung verbessern können. Aktuell gibt es etwa 10.000 Studierende in den pflegewissenschaftlichen Studiengängen und circa 1.500 Absolventen jährlich. Ein Potenzial, das zu wenig genutzt wird. In Berlin erläuterten Wissenschaftler, weshalb akademisierte Pflegekräfte besser in die Patientenversorgung integriert werden sollten.

In einer Ausgangsanalyse beschrieb Prof. Renate Stemmer, Katholische Hochschule Mainz und Vorsitzende der Deutschen Gesellschaft für Pflegewissenschaft, was eine Integration akademisch ausgebildeter Pflegemitarbeiter in die Gesundheitsversorgung jetzt zum Gebot der Stunde macht: Bedarfe generieren sich aus dem demogra- phischen Wandel, aus zunehmender Urbanisierung sowie aus Veränderungen des Krankheitsspektrums.

Multimorbide Patienten und Menschen mit chronischen Erkrankungen bedürfen einer differenzierten Pflege und Betreuung. Untersuchungen belegen eine verbesserte Patientenversorgung durch hochschulisch ausgebildete Pflegende, die wissenschaftliches Denken in das pflegerische Handeln einbeziehen. „Um spürbare Auswirkungen dieser Kompetenzen auf die Versorgungsqualität zu erreichen, ist allerdings ein quantitativer Schwellenwert in der Praxis erforderlich", so Stemmer. Die Absolventen würden zwar viele berufliche Optionen finden, aber viel zu selten in der direkten Patientenversorgung eingesetzt.

Alle Akteure im Gesundheitswesen zusammenzubringen ist nach Ansicht von Prof.
Petra Weber, Vorstand der Dekanekonferenz Pflege, unerlässlich. Sie fordert einen Diskurs zwischen Hochschulen, Politik und Unternehmen. Klientennahe Tätigkeiten seien in den Unternehmen noch zu wenig entwickelt. "Insbesondere Menschen mit chronischen Krankheitsverläufen profitieren von akademisch qualifizierten Pflegenden, weil ihre Handlungsperspektive die medizinische Seite und die individuelle Lebenssituation der betroffenen Menschen verbindet" so Weber.

(ne)

Pressekonferenz „Die Zukunft der Gesundheitsversorgung - der Beitrag akademisierter Pflegekräfte“; Berlin 5. November 2015 (Veranstalter: Deutsche Gesellschaft für Pflegewissenschaft e.V. und Dekanekonferenz Pflegewissenschaft e.V.)

\section{Hier steht eine Anzeige.}

\section{Springer}

